\title{
Wind Loading Analysis and Strategy for Deflection Reduction on HET* Wide Field Upgrade
}

\author{
Brian J. South $^{\mathrm{a}}{ }^{\dagger}$, Ian M. Soukup ${ }^{\mathrm{a}}$, Michael S. Worthington ${ }^{\mathrm{a}}$, Joseph J. Zierer ${ }^{\mathrm{a}}$, John A. Booth ${ }^{\mathrm{b}}$, \\ John M. Good \\ ${ }^{a}$ Center for Electromechanics, The University of Texas, 1 University Station R7000, \\ Austin, TX, USA 78712 \\ ${ }^{\mathrm{b}}$ McDonald Observatory, The University of Texas, 1 University Station C1402, \\ Austin, TX, USA 78712
}

\begin{abstract}
Wind loading can be a detrimental source of vibration and deflection for any large terrestrial optical telescope. The Hobby-Eberly Telescope ${ }^{*}$ (HET) in the Davis Mountains of West Texas is undergoing a Wide Field Upgrade (WFU) in support of the Dark Energy Experiment (HETDEX) that will greatly increase the size of the instrumentation subjected to operating wind speeds of up to $20.1 \mathrm{~m} / \mathrm{s}(45 \mathrm{mph})$. A non-trivial consideration for this telescope (or others) is to quantify the wind loads and resulting deflections of telescope structures induced under normal operating conditions so that appropriate design changes can be made. A quasi-static computational fluid dynamics (CFD) model was generated using wind speeds collected on-site as inputs to characterize dynamic wind forces on telescope structures under various conditions. The CFD model was refined until predicted wind speed and direction inside the dome agreed with experimental data. The dynamic wind forces were then used in static loading analysis to determine maximum deflections under typical operating conditions. This approach also allows for exploration of operating parameters without impact to the observation schedule of the telescope. With optimum combinations of parameters (i.e. dome orientation, tracker position, and louver deployment), deflections due to current wind conditions can be significantly reduced. Furthermore, the upper limit for operating wind speed could be increased, provided these parameters are monitored closely. This translates into increased image quality and observing time.
\end{abstract}

Keywords: Center for Electromechanics, Hobby-Eberly, HET, wide field upgrade, HETDEX, wind loading, deflections, louver

\section{INTRODUCTION}

The Hobby-Eberly Telescope (HET), an Arecibo astronomical telescope, is the largest at The University of Texas McDonald Observatory ${ }^{1}$. Located atop Mt. Fowlkes in the Davis Mountain Range of West Texas, the HET has an 11 meter primary mirror composed of 91 one meter hexagonal segments with 77.6 square meters of collecting area ${ }^{1,2}$. The telescope has a fixed elevation angle of $55^{\circ}$ and can be rotated $360^{\circ}$ in azimuth to cover $70 \%$ of the visible night sky ${ }^{3}$. The spherical primary mirror of the HET remains fixed during observation while a tracker system (Figure 1) moves the Prime Focus Instrument Package (PFIP) along the focal surface to track the apparent motion of celestial objects ${ }^{1,4}$. The tracker spans the upper hexagonal plane (termed "upper hex") of the metal telescope structure which also provides support for the primary mirror and is responsible for azimuth rotations. ${ }^{4}$. A dual linear drive system provides $\mathrm{X}$ and $\mathrm{Y}$ travel while a six-axis motion system controls the PFIP alignment with the focal surface ${ }^{2}$. The queued scheduling of the HET, integral to its design, provides for a distinct advantage for time-sensitive astronomy due to a rapid response to targets of opportunity ${ }^{3}$. Seen in Figure 1, the dome surrounding the telescope is an 86 foot diameter, 30 ton geodesic aluminum space frame capable of protecting the telescope from the weather of West Texas ${ }^{4}$. In-depth descriptions of the construction of the original HET telescope can be found in references 1-4.

\footnotetext{
† b.south@cem.utexas.edu; phone 1-512-232-8081; http://www.utexas.edu/research/cem/

* The Hobby-Eberly Telescope is operated by McDonald Observatory on behalf of The University of Texas at Austin, Pennsylvania State University, Stanford University, Ludwig-Maximillians-Universität München, and Georg-August-Universität, Göttingen

${ }^{*}$ http://hetdex.org/
} 
The telescope is currently undergoing a major upgrade in support of the HET Dark Energy Experiment (HETDEX) ${ }^{3,5,6}$. This upgrade will consist of replacing the current spherical aberration corrector (SAC) with a wide field corrector (WFC) as well as a new PFIP and tracker ${ }^{3}$. These improvements, referred to as the Wide Field Upgrade (WFU), will increase the field of view from 4 arcminutes to 22 arcminutes and feed an array of up to 192 new spectrographs collectively referred to as the Variable Integral-field Replicable Unit Spectrograph (VIRUS) ${ }^{5,6,7}$. These upgrades will allow for simultaneous observation of celestial objects and promote the first wide-area surveys of the emission-line universe ever conducted $^{3}$. Additional details concerning the WFU and VIRUS can be found in references 10-25.
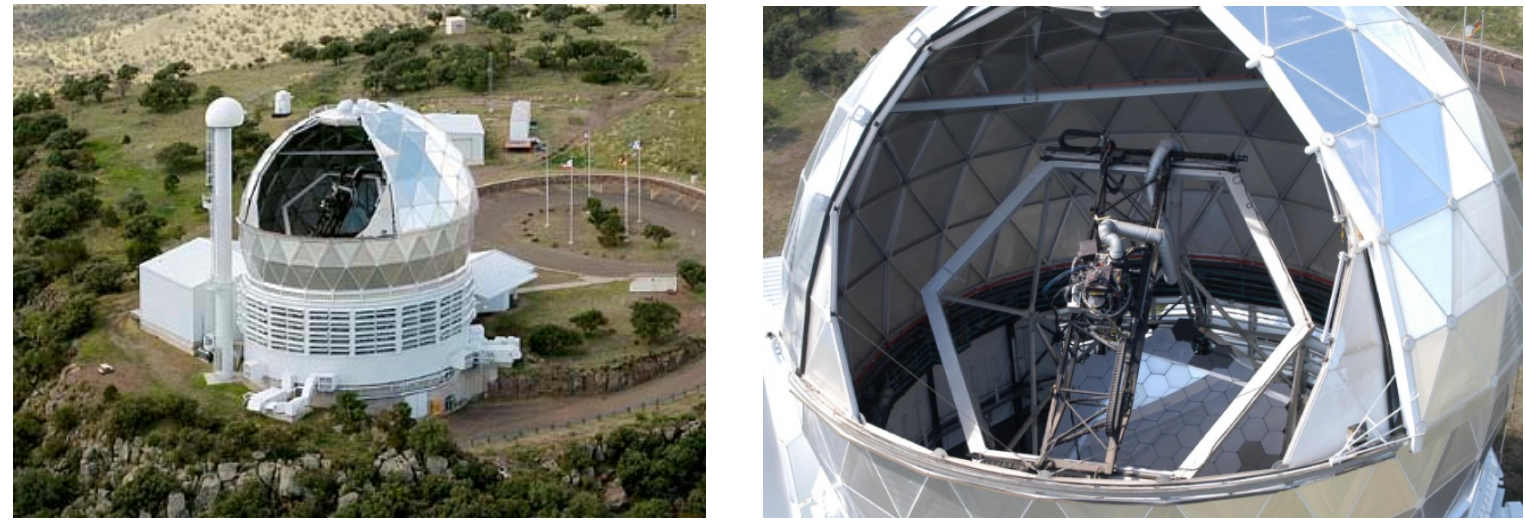

Figure 1. The photograph on the left is an aerial view of the HET telescope with the dome shutter and louvers open. The figure on the right shows the current HET tracker atop the hexagonal support frame ("upper hex").

Critical to the performance of any large telescope used for scientific studies is the integrity of the collected data. Astronomical instrumentation designers go to great lengths to preserve the throughput of the instrument by mitigating sources of obscuration, vibration, thermal expansion, etc. The forces exerted on the HET telescope structure and PFIP due to the West Texas wind are one considerable and variable source of instrument deflection. To quantify these effects, wind loading forces and resulting deflections were determined using a computational fluid dynamics (CFD) model and quasi-static loading analysis, respectively. The CFD model was validated against experimental data collected at the observatory to ensure accuracy. Through this framework, a powerful tool has been developed for optimizing the operating parameters of the telescope without impact to the observation schedule. This allows for improved data and image quality as well as an improvement to the available observing time.

\section{METHODOLOGY}

\subsection{Model generation}

To accurately recreate the winds inside the dome a full-size virtual model of the entire HET observatory was created using SolidWorks ${ }^{\circledR}$ Office Premium which allowed for easy integration with the companion CFD analysis package SolidWorks ${ }^{\circledR}$ Flow Simulation. The simplified CFD model, shown in Figure 2, was derived from the working designs for the WFU. The major structures of the tracker included the bridge, lower hexapod frame (LHF), strongback, PFIP with WFC, rho-stage, all actuators and struts as well as an early concept for the cable handlers. Models were also generated for the dome (with louvers and aperture), the upper hex frame, pier and primary mirror. To obtain appropriate meshing and limit computation time, the geometry of all parts was simplified appropriately by ignoring hardware (i.e. nuts, bolts) and complex or small features. Additionally, the telescope structure supporting the upper hex frame, primary mirror truss, catwalk, electrical cabinets, and JLG ${ }^{\circledR}$ aerial work platform were also omitted. Because the positioning and concepts for the VIRUS support structure and fiber management system was still under review, incorporation of these designs was saved for future studies. The complete model of the HET observatory used in the CFD analysis is shown in Figure 3.

\subsection{Analysis conditions}

A variety of conditions were necessary for the CFD analysis to obtain a good sample array of common observing situations. After some investigational testing of the model, a total of nine conditions were selected as a sample set. This included a combination of three dome orientations relative to wind speed (Figure 4) and three PFIP positions in the Y 
direction (Figure 5). The dome orientations included the aperture facing into the wind, aperture $45^{\circ}$ to wind direction and aperture perpendicular the wind direction. The PFIP positions were high (maximum positive Y travel), centered and low (maximum negative $\mathrm{Y}$ travel). The $\mathrm{Y}$ position was shown to have greater influence on wind loading so adjustments to the $\mathrm{X}$ position of the PFIP were omitted from this study.
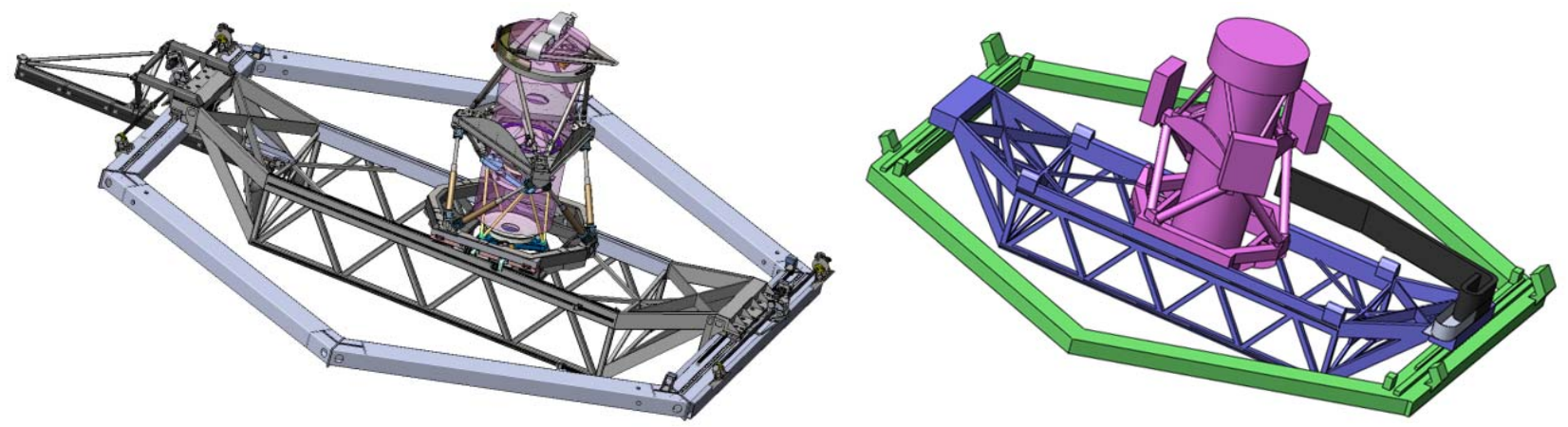

Figure2. Comparison of full size virtual design model and simplified geometry used for wind loading analysis.
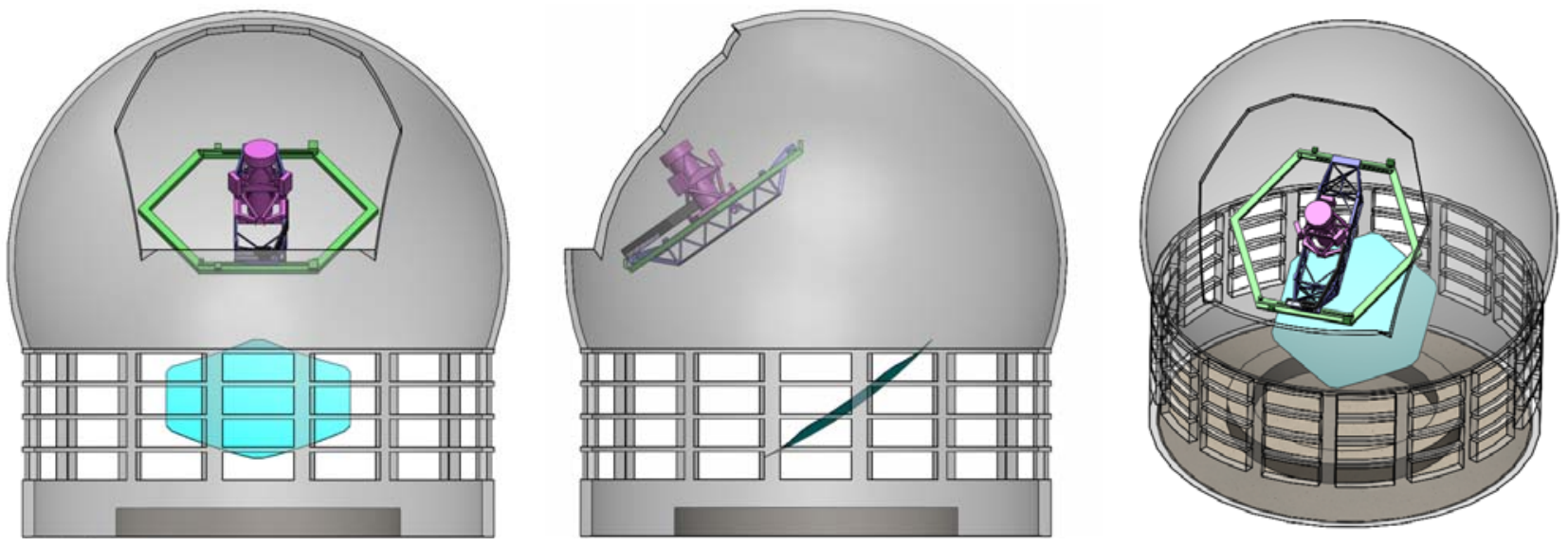

Figure 3. Complete model of future HET observatory used in CFD analysis. This model includes the bridge, lower hexapod frame (LHF), strongback, PFIP/WFC, rho-stage, actuators, struts, cable handlers, upper hex frame, pier, primary mirror, and dome.
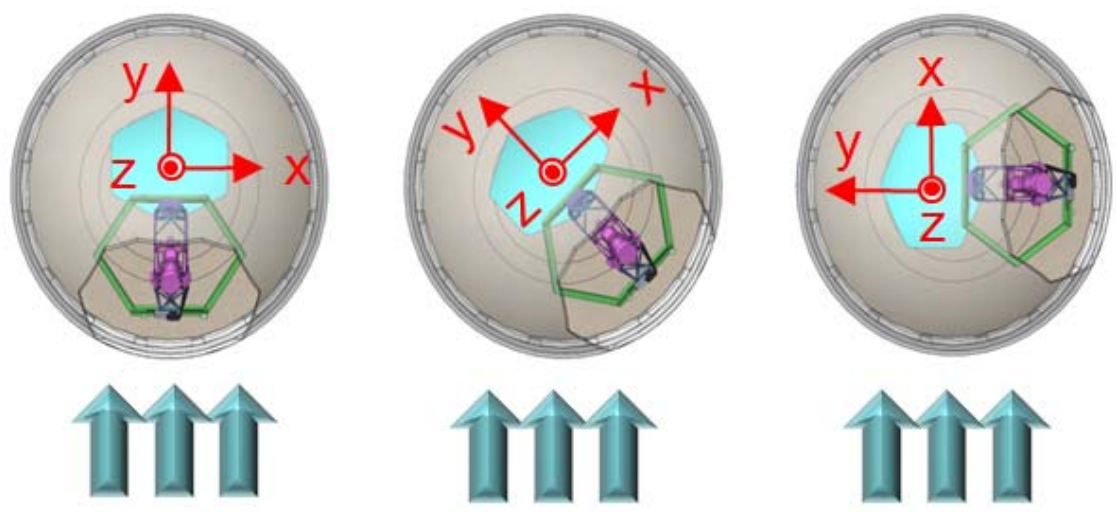

Figure 4. The three wind directions used in wind loading analysis. The blue arrows indicate the wind direction relative to dome orientation. Left-to-right: aperture facing wind; aperture 45 degrees to wind and aperture perpendicular to wind. 

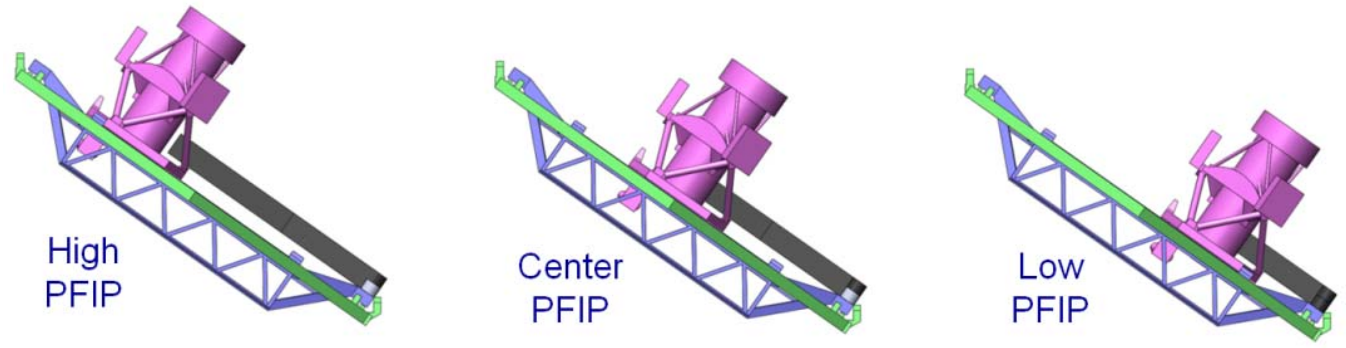

Figure 5. The three PFIP positions used in wind loading analysis. Left-to-right: high PFIP; centered PFIP and low PFIP. High and low PFIP positions are at the maximum positive and negative Y travel, respectively

\subsection{Analysis parameters and meshing}

Parameters for the CFD analysis were selected to match the on-site environmental conditions at the HET observatory. The air was dry at a temperature of $20^{\circ} \mathrm{C}\left(68^{\circ} \mathrm{F}\right)$. An atmospheric pressure of $101,325 \mathrm{~Pa}(1 \mathrm{~atm})$ was used and the analysis assumed adiabatic wall conditions. Both laminar and turbulent flow were considered and internal cavities were excluded. The external wind speed was set to $20.1 \mathrm{~m} / \mathrm{s}(45 \mathrm{mph})$ which corresponds to the HET's acceptable peak gust under normal operating conditions ${ }^{8}$.

The computational domain was set to 80 meters wide, 160 meters long and 62 meters tall in the global $\mathrm{X}, \mathrm{Y}$, and $\mathrm{Z}$ directions, respectively. For ease of transition and uniformity of analysis conditions, the wind direction was always along the $\mathrm{Y}$ axis and the telescope model was rotated about its azimuth as needed. The total volume of 793,600 cubic meters ensured all airflow effect above, behind and to the sides of the dome were captured.

Flow Simulation utilizes an initial mesh, which included over 500,000 cells, and an automatic narrow channel refinement up to $2 \mathrm{~cm}$. The total final mesh contained over 2.7 million cells. The mesh refinement of the computational domain is shown in Figure 6.

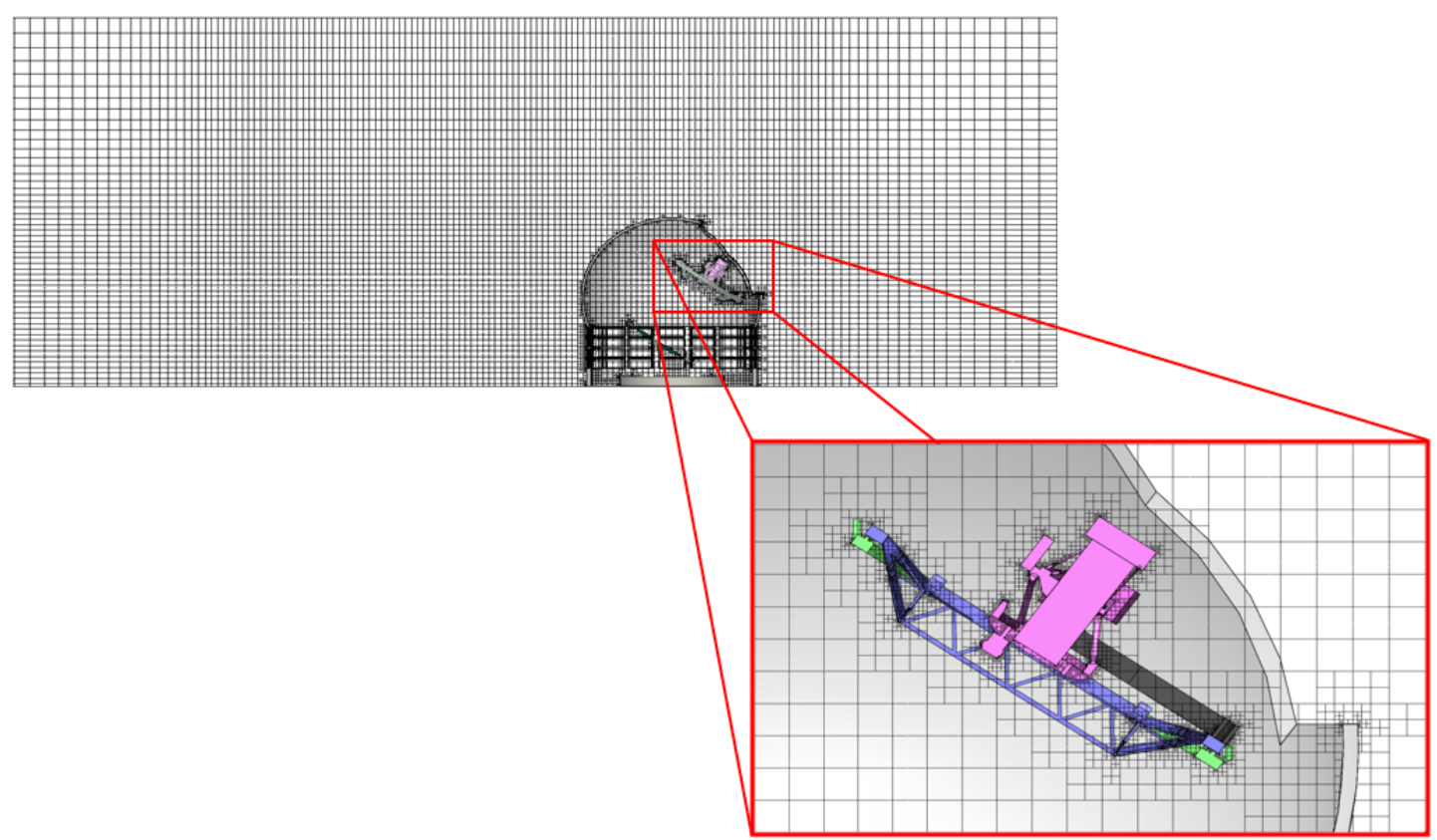

Figure 6. Meshing of entire computational domain for analysis showing small feature refinement. 


\section{RESULTS}

\subsection{Qualitative observations}

As shown in Figure 7, the HET observatory creates large wind disturbances in-front and behind the dome. As observed on-site ${ }^{9}$, the wind velocities inside the dome can vary considerably as a function of wind direction and PFIP position. When the dome aperture is oriented facing the wind (Figure 8, left), the wind speed drops off considerably just before reaching the PFIP. When the wind is dome is turned to $45^{\circ}$, a counterclockwise circular flow pattern is created (Figure 8 , middle). Finally, when the dome aperture is perpendicular to the wind direction, most of the instrumentation is shielded from the high winds (Figure 8, right).
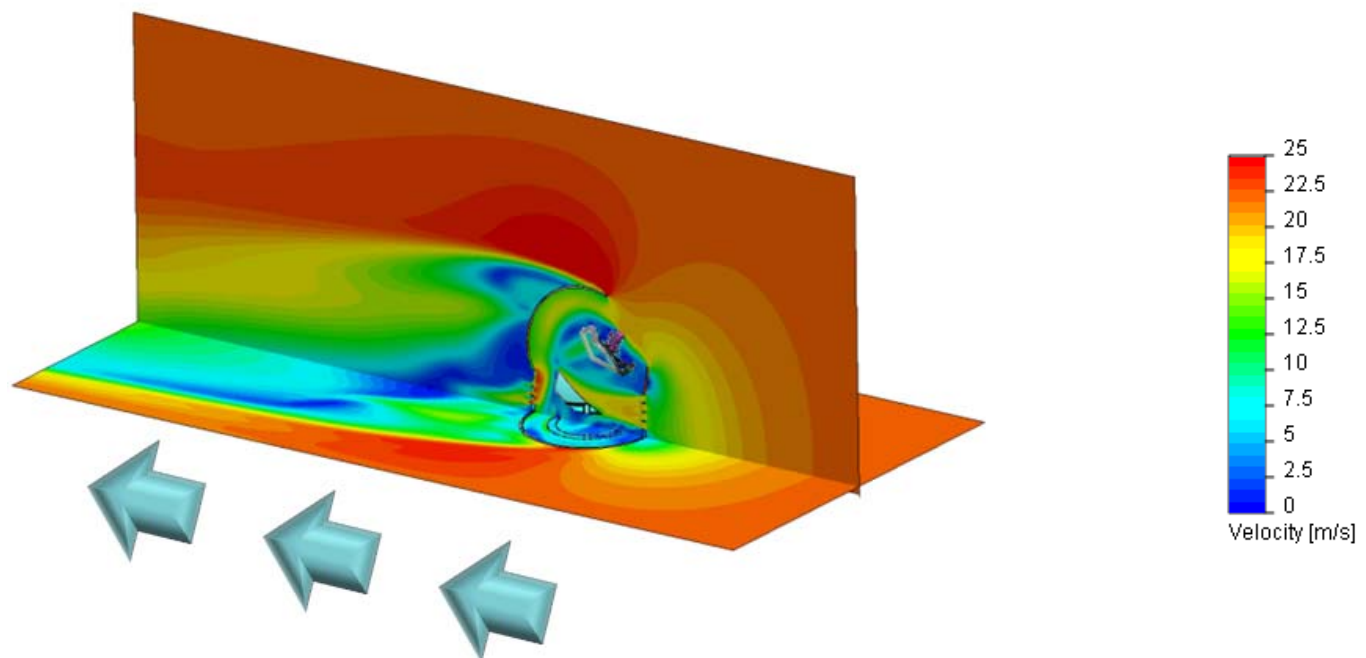

Figure 7. Dual section views showing three dimensional effects of wind circulating in and around dome. Blue arrows indicate wind direction. The configuration shown is the dome aperture facing into the wind with a centered PFIP.
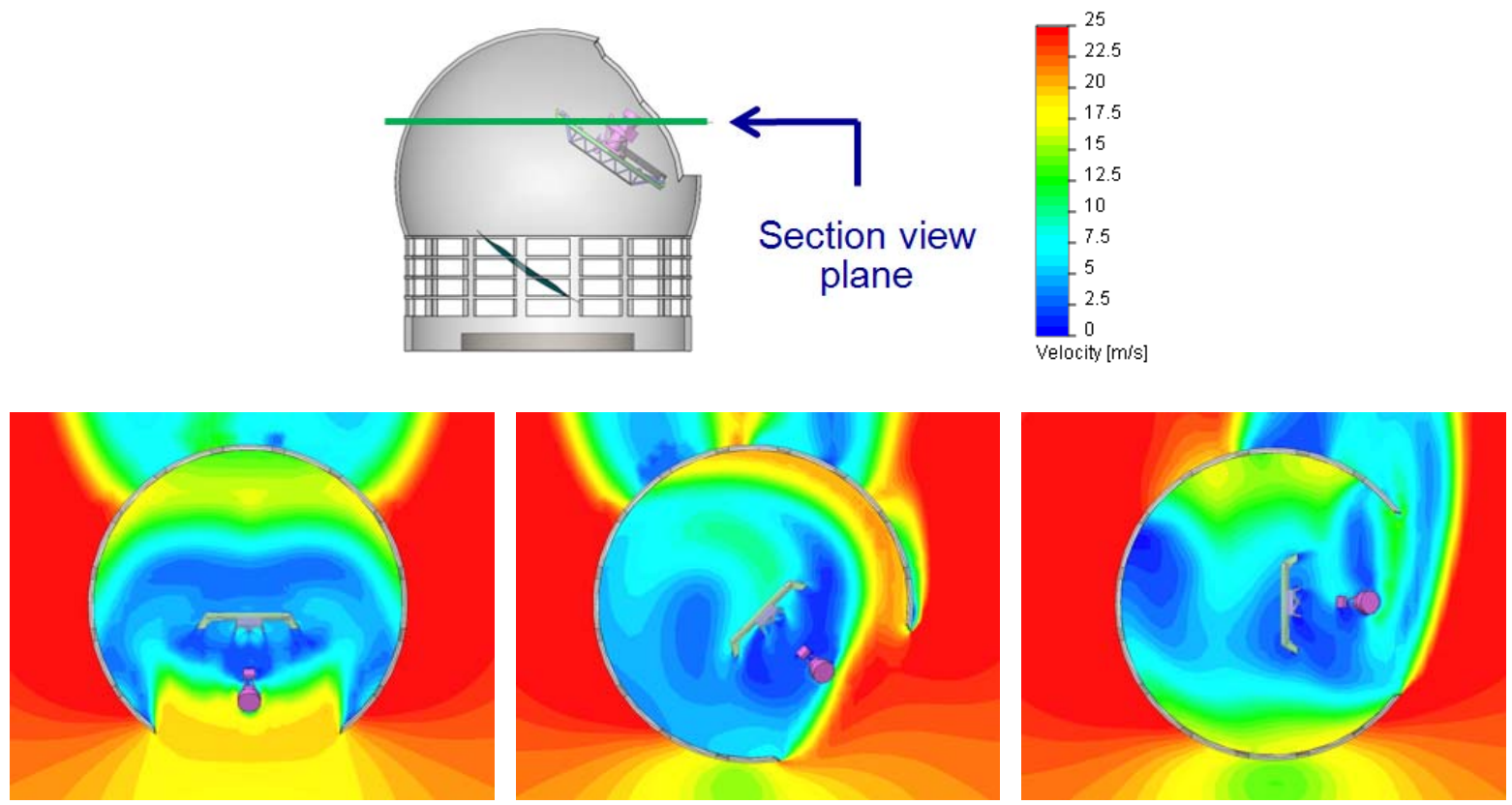

Figure 8. Section view wind velocity contour plots of three dome orientations (left-to-right): aperture facing wind, aperture $45^{\circ}$ to wind, aperture perpendicular to wind. The wind direction is from bottom to top in all three figures. 
Figure 9 provides side-by-side comparisons of the wind velocities generated inside the dome for all nine conditions analyzed. Notice that when the dome aperture is facing the wind (Figure 9, left column), airflow is directed over the top of the PFIP and out the back via the louvers. Also, at an aperture angle of 45 degrees, the low PFIP position (Figure 9, bottom middle) places the instrumentation closer to the path of the high velocity winds. Finally, all three PFIP positions at the perpendicular shutter angle (Figure 9, right column) keep the instrumentation safely away from high velocity airflow.

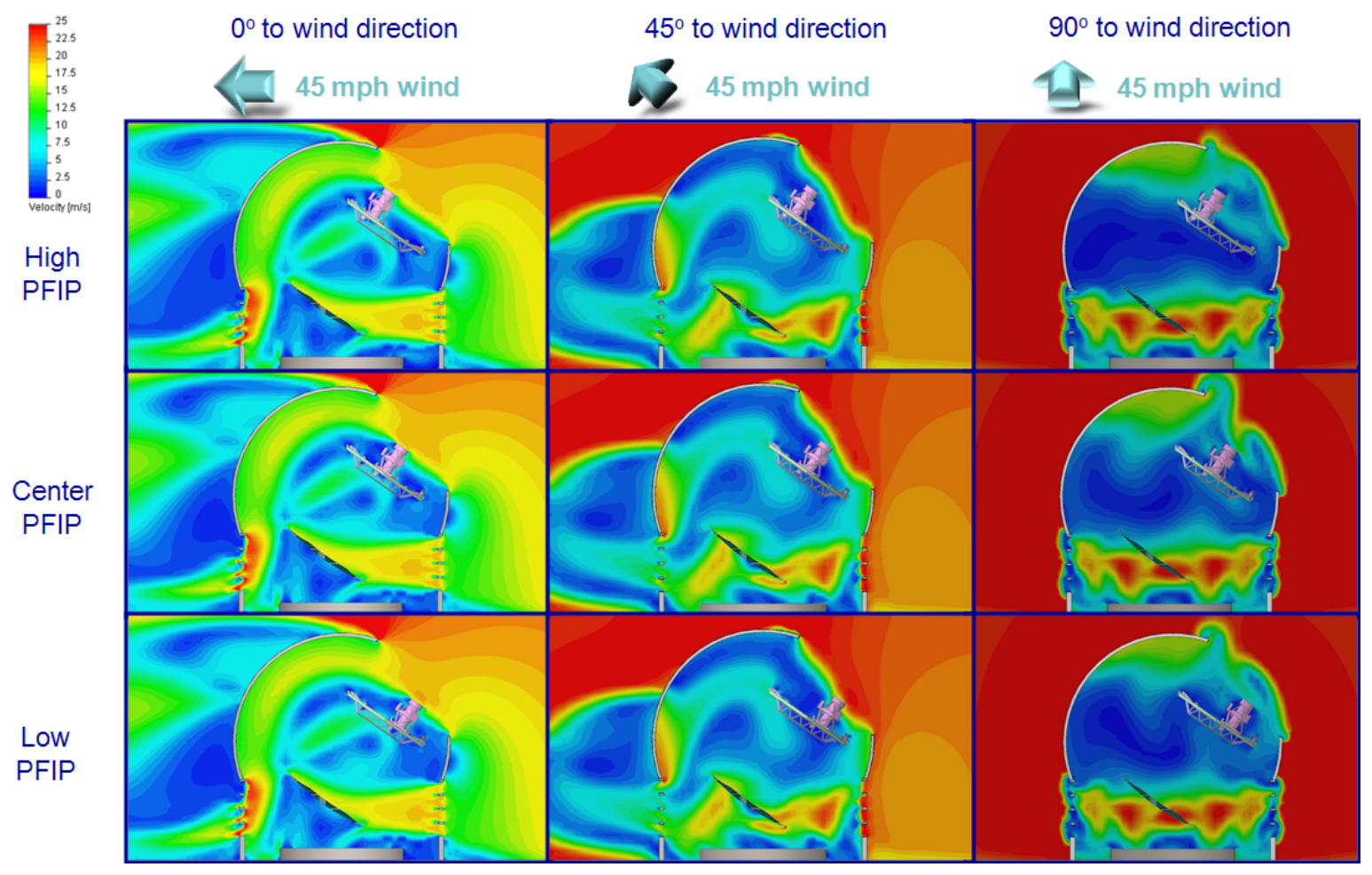

Figure 9. Section view comparison of wind velocity contour plots of the nine configurations studied. Note that each section plane bisects the telescope in the Y-Z plane (global) while the wind direction is varied.

\subsection{Pressure versus velocity}

Similar to air flow over the wing of an aircraft, the curvature of the HET dome creates a relationship between the pressure and speed of the air flow. As seen in Figure 10, the higher air speed corresponds to a lower pressure and viceversa. The dome helps to buffer the high wind speed by generating higher pressures inside and in front of the dome.

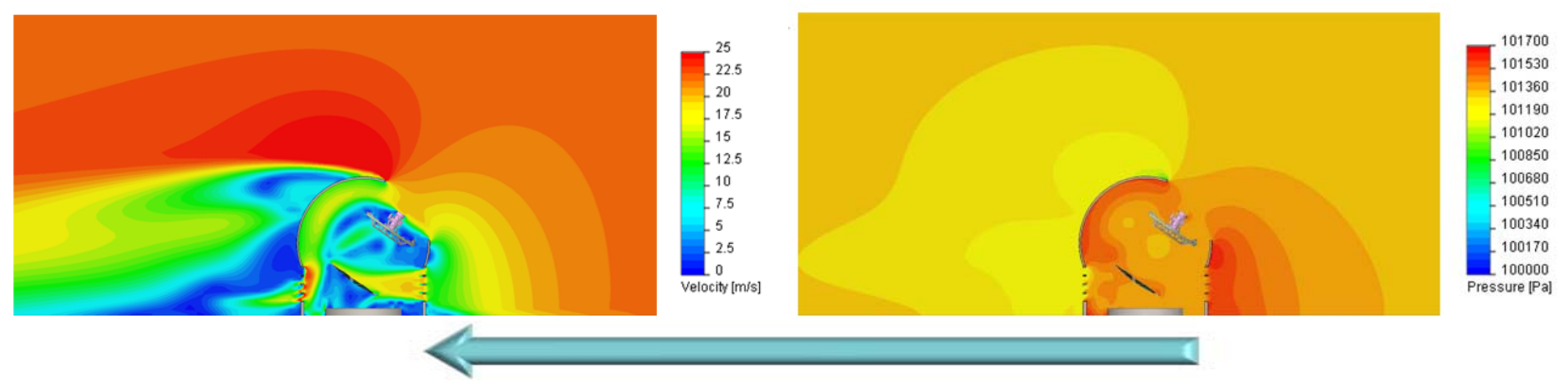

Figure 10. Comparison between wind speeds (left) and air pressure (right). Both figures are of the same analysis condition: dome aperture into wind with centered PFIP. The blue arrow indicates wind direction in both views. 


\subsection{Data Validation}

The overall results of the CFD analysis correspond exceedingly well with qualitative observations made of wind flows inside the HET dome 9 . To gain further confidence in the validity of the study, quantitative measurements were made of wind speeds on-site using R. M. Young Company Model 81000 Ultrasonic Anemometers, capable of collecting three dimensional wind velocities. Two of these anemometers were installed on the corners of the upper hex at the furthest possible positive and negative X position. During the on-site data collection, measurements were taken of the tracker position, dome orientation and external wind speed. The external wind direction was only known relative to the azimuth angle of the dome so only the magnitudes of the wind velocity were used for comparison purposes. Unfortunately due to frequently shifting West Texas winds, a steady-state relationship between the external and internal wind speeds was implied with a limited confidence. Once the on-site data was collected, an additional simulation of the CFD model was created which matched the empirical conditions so that an accurate comparison could be made. The first anemometer recorded a wind magnitude of $2.6 \pm 1.2 \mathrm{~m} / \mathrm{s}$ corresponding to a CFD prediction of $1.17 \mathrm{~m} / \mathrm{s}$ while the second recorded $1.9 \pm 0.4 \mathrm{~m} / \mathrm{s}$ with a CFD prediction of $1.93 \mathrm{~m} / \mathrm{s}$. Considering the frequently shifting West Texas winds, which make correlating a steady-state relationship between external and internal wind speed difficult, these results show a remarkable similarity and instill confidence in the CFD model's ability to predict wind flow patterns in the HET observatory.

\subsection{Wind loading forces and resulting deflections}

The primary convergence criteria for the CFD analysis was the $\mathrm{X}, \mathrm{Y}$ and $\mathrm{Z}$ forces exerted on each major substructure of the telescope. The major components considered were the bridge, LHF, and PFIP. Figure 10 and Table 1 provide a breakdown of these forces for each wind loading condition. The highest single load predicted was the $\mathrm{X}$ force on the PFIP while in a low position and $45^{\circ}$ to the wind direction (discussed later). However, the condition with the highest combined forces (worst case) was the dome facing into the wind with a high PFIP location. Using similar Finite Element Analysis (FEA) techniques to those described in refs 10 and 11, these loads were then used in a static loading analysis of the telescope structure to yield a combined total deflection of $8.34 \mu \mathrm{m}(\delta \mathrm{X}=-1.05 \mu \mathrm{m}, \delta \mathrm{Y}=7.96 \mu \mathrm{m}, \delta \mathrm{Z}=$ $2.22 \mu \mathrm{m}$ ) at the rho-stage focal surface assembly (uppermost structure of the PFIP).

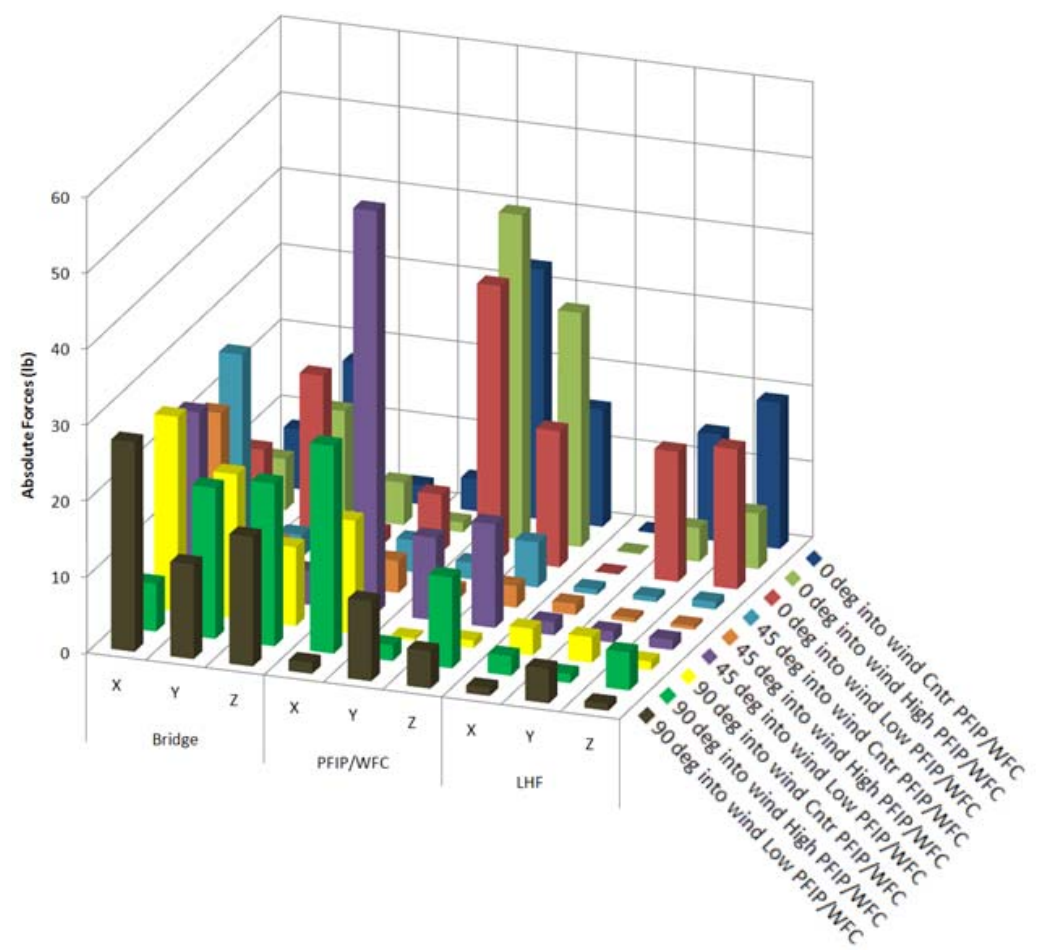

Figure 10. Wind loading forces on each major substructure for the nine analyzed conditions. All forces are in telescope coordinates. 
Table 1. Wind loading forces on each major substructure for the nine analyzed conditions. All forces are in telescope coordinates and the units are lbs.

\begin{tabular}{|c|c|c|c|c|c|c|c|c|c|}
\hline & \multicolumn{3}{|c|}{ Bridge } & \multicolumn{3}{|c|}{ PFIP/WFC } & \multicolumn{3}{|c|}{ LHF } \\
\hline & $\mathrm{x}$ & Y & z & $\mathrm{x}$ & Y & z & $\mathrm{x}$ & Y & z \\
\hline 0 deg into wind Cntr PFIP/WFC & 8 & 18 & 3 & 4 & 33 & 15 & 0 & 14 & 19 \\
\hline 0 deg into wind High PFIP/WFC & 7 & 14 & 6 & 1 & 43 & 31 & 0 & 4 & 7 \\
\hline 0 deg into wind Low PFIP/WFC & 11 & 21 & 2 & 8 & 36 & 18 & 0 & 17 & 18 \\
\hline I 45 deg into wind Cntr PFIP/WFC & 26 & 3 & 6 & 4 & 2 & 6 & 1 & 1 & 1 \\
\hline I5 deg into wind High PFIP/WFC & 21 & 5 & 5 & 4 & 1 & 3 & 1 & 1 & 0 \\
\hline - 45 deg into wind Low PFIP/WFC & 24 & 1 & 4 & 53 & 11 & 14 & 2 & 1 & 1 \\
\hline 90 deg into wind Cntr PFIP/WFC & 26 & 19 & 10 & 15 & 0 & 1 & 4 & 3 & 1 \\
\hline - 90 deg into wind High PFIP/WFC & 6 & 20 & 21 & 27 & 2 & 12 & 3 & 1 & 5 \\
\hline 90 deg into wind Low PFIP/WFC & 28 & 12 & 17 & 1 & 11 & 5 & 1 & 5 & 1 \\
\hline
\end{tabular}

\section{DISCUSSION}

\subsection{Investigation of irregularity}

A sizeable advantage of utilizing a CFD analysis is the ability to take a close look at any detail of the results. For example, one seemingly anomalous wind load was the X direction load on the PFIP while positioned low on the Y axis and at a dome aperture angle of $45^{\circ}$ to the wind direction (large purple bar in Figure 10). Upon further investigation of appropriate flow lines, it was determined that in this particular orientation, high speed winds are entering the dome in the same volume occupied by the top of the PFIP. As seen in Figure 11, the low PFIP is placed directly in the path of these high speed winds (right) while the centered PFIP remains protected (left). This is an important implication since it can indicate which tracker positions to avoid under a given set of circumstances. By incorporating these guidelines into the queued scheduling of the HET, much greater image and data quality can be achieved. Furthermore, through additional analysis, the maximum operating wind speed may be increased as long as the telescope operator pays careful attention to the tracker position and external wind direction. This would allow for increased observation time and also integrates well with HET's queue schedule. Additionally, exploration of these innovative operation parameters can be conducted via simulation without any risk of damage to the instrumentation mounted on the telescope.
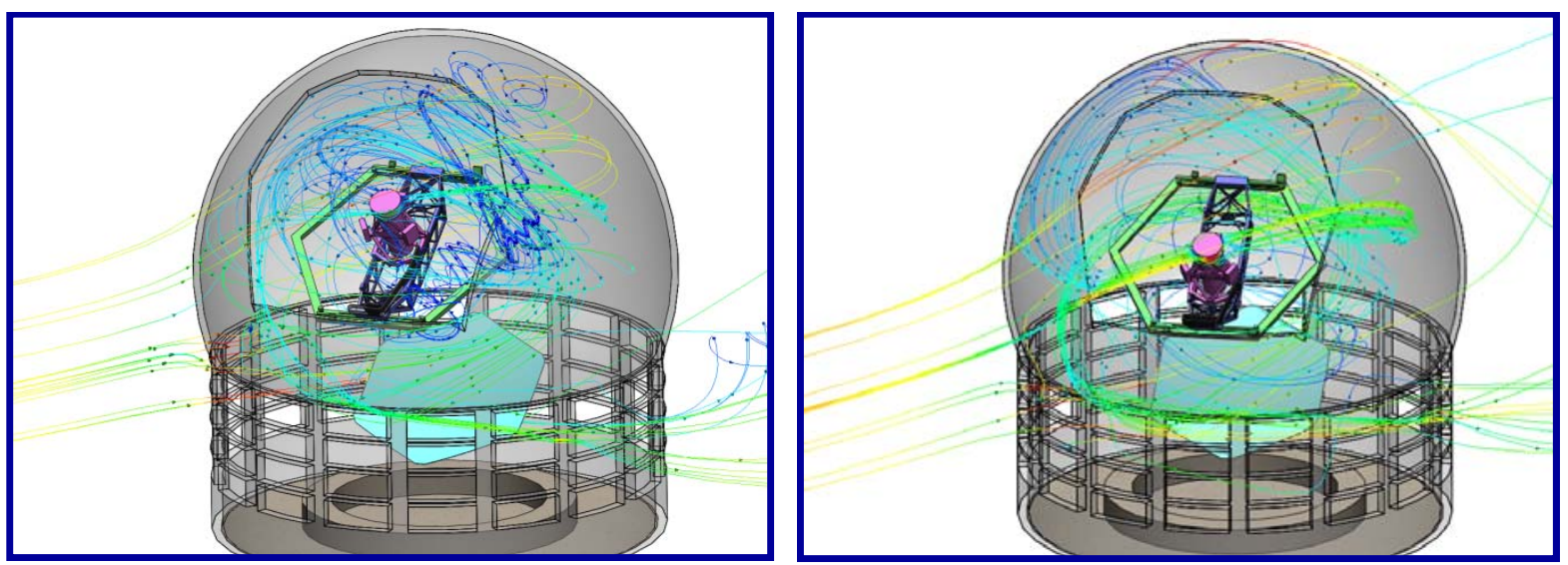

Figure 11. Traces of wind velocity jet stream entering dome. The figure on the left is a centered PFIP placed 45 degrees to wind direction while the figure on the right is a low PFIP with the same wind direction. These plots show how the placement of the PFIP can greatly affect the forces experienced by the instrumentation.

\subsection{Selective louver deployment}

By observing the airflow patterns produced by the simulation, many concepts for reducing the deflections due to the wind can be explored. One suitable approach is the selective deployment of individual louvers below the rotating dome 
of the telescope. Originally installed to aid in ventilation and mitigate thermal effects ${ }^{25}$, these louvers have a substantial influence on the airflow patterns inside the dome. An initial investigation showed that by simply closing the three columns of louvers opposite the dome aperture when facing into the wind, a $50 \%$ reduction in wind loads can be achieved (Figure 2, middle). However, closing both the front and rear three columns of louvers under the same conditions dramatically increases the loads seen on the instrumentation by creating high velocity vortex airflow inside the dome (Figure 12, bottom). This highlights the importance of careful evaluation of operating parameters before implementation.

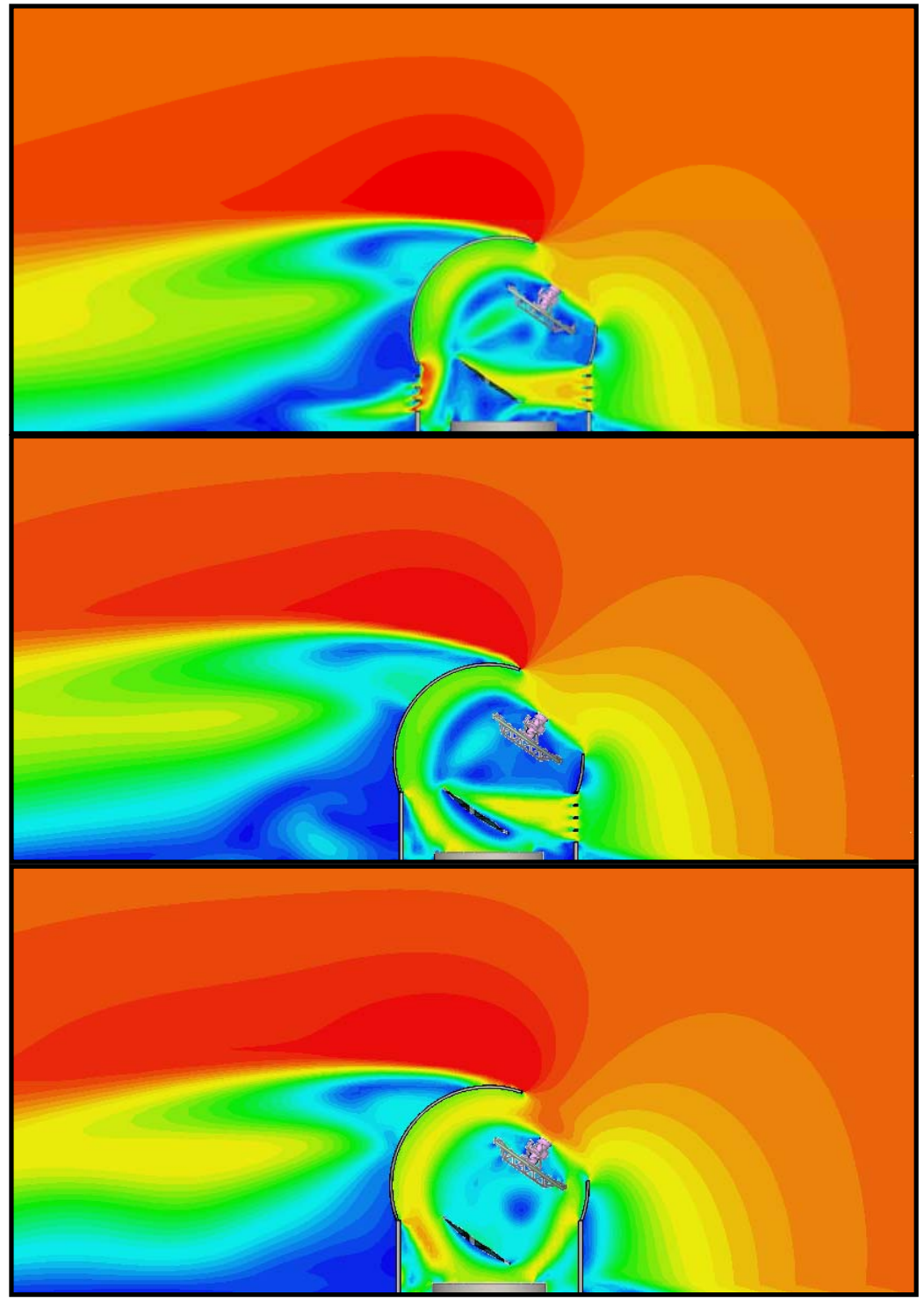

Figure 12. Cross section velocity contour plots to demonstrate the impact of selectively deploying the louvers of the HET dome. The top figure is the nominal condition with all the dome louvers open. In the middle figure, the three columns of louvers opposite the dome aperture are closed, dramatically reducing the loads seen on the telescope. The bottom figure shows the very unfavorable vortex airflow pattern that results from closing the front and rear three louvers on the telescope. Blue arrow indicates wind direction in all three figures. 


\subsection{Caveats and future work}

The results presented in this study are the first attempt to quantify the loads and deflections induced on the HET telescope. Like any simulation, however, the real world values may vary. The CFD model will continue to need refinement and further evaluation to be an acceptably accurate and effective load prediction tool. Specifically, some of the latest designs (i.e. IFU, VIRUS enclosure) are not included in the present study. Likewise, many building features were omitted from the study which can influence the results (though likely only a small deviation). Future work should also focus on investigating additional wind speeds and operating conditions to get a complete picture of the complex interactions of the wind. Finally, the influence of thermal effects and temperature gradients can easily be included into CFD analysis as simply another parameter when creating the study.

\section{SUMMARY}

Overall, the CFD analysis methodology outlined in this study proved to be extremely powerful tool for analyzing the airflow behavior and effects of wind loading on the HET observatory. The analysis results were shown to be accurate, matching up well with both qualitative observations and quantitative on-site measurements. Investigation of novel operating parameters such as selective louver deployment or combinations of dome aperture/PFIP orientations can be performed without impact to the normal operations or scheduling of the telescope. Simulation results can be iterated upon repeatedly until satisfactory confidence is achieved prior to implementation on the telescope. Furthermore, the ability to easily extract the forces exerted on the telescope structure due to the wind is also an important guideline for reducing deflections in telescope structural design. All of these advantages directly translate into better management of operating parameters, increased observation time, improved data quality and better overall science.

\section{ACKNOWLEDGEMENTS}

HETDEX is led by the University of Texas at Austin McDonald Observatory and Department of Astronomy with participation from the Universitäts-Sternwarte of the Ludwig-Maximilians-Universität München, the Max-PlanckInstitut für Extraterrestrische-Physik (MPE), Astrophysikalisches Institut Potsdam (AIP), Texas A\&M University, Pennsylvania State University, and the HET consortium. In addition to Institutional support, HETDEX is funded in part by gifts from Harold C. Simmons, Robert and Annie Graham, The Cynthia and George Mitchell Foundation, Louis and Julia Beecherl, Jim and Charlotte Finley, Bill and Bettye Nowlin, Robert and Fallon Vaughn, Eric Stumberg, and many others, by AFRL under agreement number FA9451-04-2-0355, and by the Texas Norman Hackerman Advanced Research Program under grants 003658-0005-2006 and 003658-0295-2007.

\section{REFERENCES}

[1] Krabbendam, V.L., Sebring, T.A., Ray, F.B. and Fowler, J.R., "Development and performance of Hobby-Eberly Telescope 11-m segmented mirror," Proc. SPIE 3352, 436-445 (1998).

[2] Booth, J. A., Ray, F. B. and Porter, D. S., "Development of a star tracker for the Hobby Eberly Telescope," Proc. SPIE 3351, 298-309 (1998).

[3] Hill, G. J., MacQueen, P. J., Palunas, P., Shetrone, M. D. and Booth, J. A., "Present and Future Instrumentation for the Hobby-Eberly," Proc. SPIE 7014, 701406 (2008).

[4] Ramsey, L.W., et al., "The early performance and present status of the Hobby-Eberly Telescope," Proc. SPIE 3352, 34-42 (1998).

[5] Booth, J. A., MacQueen, P. J., Good, J. M., Wesley, G. L., Hill, Palunas, P., Segura, P. R. and Calder, R. E., "The wide field upgrade for the Hobby-Eberly Telescope", Proc. SPIE 6267, 62673W (2006).

[6] Savage, R., et al., "Current Status of the Hobby-Eberly Telescope wide field upgrade," Proc. SPIE 7733-149 (2010)

[7] Heisler, J. T., et al., "Integration of VIRUS spectrographs for the HET dark energy experiment," Proc. SPIE 7733153 (2010).

[8] Projects Document HX0005. "HETDEX and HET Wide Field Upgrade Technical Requirements"

[9] Good, J. M., "Characterization of Flow in the HET Enclosure Following Ventilation and Application of Lowemissive Coatings." The University of Texas at Austin McDonald Observatory Internal Document.

[10] Worthington, M. S., Nichols, S. P., Good, J. M., Zierer, J. J., Mollison, N. T. and Soukup, I. M., "Design and analysis of the Hobby-Eberly Telescope dark energy experiment (HETDEX) bridge," Proc. SPIE 7733-147 (2010). 
[11]Zierer, J. Z., Mock, J. R., Beno, J. H., Lazzarini, P. G., Fumi, P., Anaclerio, V. and Good, J. M., "The development of high-precision hexapod actuators for the Hobby-Eberly Telescope Dark Energy Experiment (HETDEX)", Proc. SPIE 7733-49 (2010).

[12] Hill, G. J., Adams, J. J., Blanc, G., Booth, J. A., Chonis, T. S., Cornell, M. E., DePoy, D. L., Drory, N., Gebhardt, K., Good, J. M., Grupp, F. U., Kelz, A., Lee, H., Marshall, J. L., MacQueen, P. J., Mollison, N. T., Murphy, J. D. and Rafal, M. D., "VIRUS: a massively replicated 33k fiber integral field spectrograph for the upgraded HobbyEberly Telescope," Proc. SPIE 7735-21 (2010).

[13] Mollison, N. T., Mock, J. R. Soukup, I. M., Beets, T. A., Good, J. M., Beno, J. H., Kriel, H. J., Hinze, S. E. and Wardell, D. R., "Design and development of a long-travel positioning actuator and tandem constant force actuator safety system for the Hobby-Eberly Telescope wide-field upgrade," Proc. SPIE 7733-150 (2010).

[14] Mock, J. R., Beno, J. H., Zierer, J. J., Rafferty, T. H. and Cornell, M. E., "Tracker controls development and control architecture for the Hobby-Eberly Telescope dark energy experiment”, Proc. SPIE 7733-152 (2010).

[15] Worthington, M. S., Beets, T. A., Beno, J. H., Mock, J. R., Murphy, B. T., South, B. J. and Good, J. M., "Design and development of a high precision, high payload telescope dual drive system," Proc. SPIE 7733-201 (2010).

[16] Hill, G. J., Gebhardt, K., Komatsu, E., Drory, N., MacQueen, P. J., Adams, J. J., Blanc, G. A., Koehler, R., Rafal, M., Roth, M. M., Kelz, A., Gronwall, C., Ciardullo, R. and Schneider, D. P., "The Hobby-Eberly Telescope Dark Energy Experiment (HETDEX): Description and Early Pilot Survey Results," ASP Conf. Series 399, 115-118 (2008).

[17] Soukup, I. M., et al., "Design of the fiber optic support system and fiber bundle accelerated life test for VIRUS," Proc. SPIE 7735-180 (2010).

[18] Beno, J. H., Mock, J. R. and Booth, J. A., “An alternative architecture and control strategy for hexapod positioning systems to simplify structural design and improve accuracy,"Proc. SPIE 7733-97 (2010).

[19] Collins, A., et al., "Development of VIRUS alignment assembly fixtures," Proc. SPIE 7735-263 (2010).

[20] Vattiat, B., et al., "Mechanical design evolution of the VIRUS instrument for volume production and deployment," Proc. SPIE 7735-264 (2010).

[21] Mollison, N. T., et al., "Collaborative engineering and design management for the Hobby-Eberly Telescope wide field upgrade," Proc. SPIE 7738-84 (2010).

[22] Ramiller, C., et al., "A new control system hardware architecture for the Hobby-Eberly Telescope prime focus instrument package," Proc. SPIE 7740-129 (2010).

[23] Good, J. M., et al., "Design of performance verification testing for HET wide-field upgrade tracker in the laboratory," Proc. SPIE 7739-152 (2010).

[24]Burge, J. H. et al, "Development of a wide-field spherical aberration corrector for the Hobby-Eberly Telescope", Proc. SPIE 7733-51 (2010).

[25] Kelz, A., Roth, M. M., Bauer, S. M., Padilla, Y., Popow, E., Hill, G. J., Vattiat, B. L., Good, J. M., Murphy, J. D., Savage, R. D., Soukup, I. M. and Mollison, N. T., "Production and performance of replicable integral field units for VIRUS", Proc. SPIE 7735-178 (2010). 